\title{
PAID-INCURRED CHAIN RESERVING METHOD WITH DEPENDENCE MODELING
}

\author{
BY \\ SEBASTIAN HAPP AND MARIO V. WÜTHRICH
}

\begin{abstract}
The paid-incurred chain (PIC) reserving method is a claims reserving method that allows to combine claims payments and incurred losses information in a mathematical consistent way. The main criticism on the original Bayesian lognormal PIC model presented in Merz-Wüthrich [5] is that it does not respect dependence properties within the observed data. In the present paper, we extend the original Bayesian log-normal PIC model so that dependence is modeled in an appropriate way.
\end{abstract}

\section{KEYWORDS}

Claims reserving, outstanding loss liabilities, ultimate loss prediction, claims payments, claims incurred, incurred losses, prediction uncertainty, paidincurred chain model, PIC reserving method.

\section{PAID-INCURRED CHAIN MODEL WITH DEPENDENCE MODELING}

\subsection{Introduction}

The process of assessing the outstanding loss liabilities is an important part of the management of a non-life insurance company. Reserving actuaries need to predict the outstanding loss liabilities based on all available information. In many cases, this available information comprises claims payments observations, incurred losses data and, may be, prior loss ratio information. State-of-the-art stochastic claims reserving methods are not able to combine these three sources of information in a mathematically consistent way. Often in practice, actuaries apply different claims reserving methods to these different sources of information. The results of these different methods are then merged in an ad hoc way to obtain one prediction of the outstanding loss liabilities. Of course, such ad hoc considerations do not allow one to assess prediction uncertainty because, typically, the merging is done subjectively by pure expert opinion. 
One of the first claims reserving techniques in the actuarial literature to unify outstanding loss liability prediction based on claims payments and incurred losses information is the Munich chain ladder (MCL) method. The MCL method was introduced by Quarg-Mack [8] and its aim is to reduce the gap between the two chain ladder (CL) predictions that are based on claims payments and incurred losses data, respectively. The idea is to adjust the CL factors with paid-incurred ratios to reduce the gap between the two predictions. The difficulty with the MCL method is that it involves several parameter estimates whose precisions are difficult to quantify within a stochastic model framework.

In this paper, we revisit the Bayesian log-normal paid-incurred chain (PIC) model of Merz-Wüthrich [5]. This PIC reserving model was motivated by Posthuma et al. [7] who have designed a similar model bringing paid-incurred information in-line. The PIC reserving method combines claims payments and incurred losses information in such a way that Bayesian inference can be made on the missing (future) part of the claims development triangles based on both sources of information, see also Figure 1. The main criticism on the models of Posthuma et al. [7] and Merz-Wüthrich [5] is that they do not respect dependence properties in an appropriate way. In the present paper, we expand the initial Bayesian log-normal PIC model such that actual dependence structure found in the data is respected.

\subsection{Notation and model assumptions}

For the PIC model, we consider three channels of information: (i) claims payments, which refer to the payments done for reported claims; (ii) incurred losses, which correspond to the reported claim amounts; (iii) prior expert opinion. The crucial observation is that the claims payments and incurred losses time series must reach the same ultimate value, because these two time series both converge to the total ultimate claim. By choosing appropriate model assumptions, we force this property to hold true in our model.

In the following, we denote accident years by $i \in\{0, \ldots, J\}$ and development years by $j \in\{0, \ldots, J\}$. We assume that all claims are settled after the $J$ th development year. Cumulative claims payments in accident year $i$ after $j$ development periods are denoted by $P_{i, j}$ and the corresponding incurred losses by $I_{i, j}$. Moreover, for the ultimate claim, we assume (force) $P_{i, J}=I_{i, J}$ with probability 1 , which means that ultimately (at time $J$ ) they reach the same ultimate value. For an illustration we refer to Figure 1.

We introduce the following notation, for $i \in\{0, \ldots, J\}$,

$$
\Xi_{i}=\left(\zeta_{i, 0} ; \zeta_{i, 1}, \xi_{i, 1}, \zeta_{i, 2}, \xi_{i, 2}, \ldots, \zeta_{i, J}, \xi_{i, J}\right)^{\prime} \in \mathbb{R}^{2 J+1},
$$

with, for $j \in\{0, \ldots, J\}$ and $l \in\{1, \ldots, J\}$,

$$
\zeta_{i, j}=\log \frac{I_{i, j}}{I_{i, j-1}} \quad \text { and } \quad \xi_{i, l}=\log \frac{P_{i, l}}{P_{i, l-1}}
$$




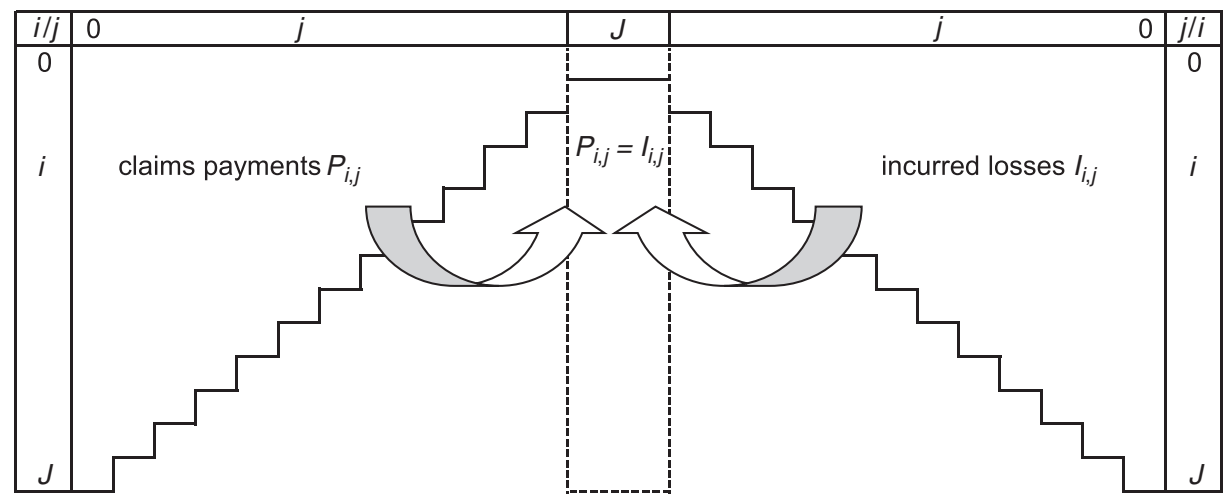

FIGURE 1: Left-hand side: cumulative claims payments $P_{i, j}$ development triangle; right-hand side: incurred losses $I_{i, j}$ development triangle; both leading to the same ultimate claim $P_{i, J}=I_{i, J}$.

where we have set $I_{i,-1} \equiv 1$. The Bayesian log-normal PIC model with dependence within the random vectors $\Xi_{i}$ is then defined as follows.

\section{Model Assumptions 1.1 (Bayesian log-normal PIC model with dependence).}

- Conditionally, given $\Theta=\left(\Psi_{0} ; \Psi_{1}, \Phi_{1}, \Psi_{2}, \Phi_{2}, \ldots, \Psi_{J}, \Phi_{J}\right)^{\prime}$ and $V \in$ $\mathbb{R}^{(2 J+1) \times(2 J+1)}$,

- the random vectors $\Xi_{0}, \ldots, \Xi_{J}$ are i.i.d. with multivariate Gaussian distribution

$$
\Xi_{i} \sim \mathcal{N}(\Theta, V) \text { for } i=0, \ldots, J
$$

- we assume $P_{i, J}=I_{i, J}$ for all $i=0, \ldots, J$, $\mathbb{P}$-a.s.

- $V$ is a positive definite covariance matrix and the components of $\Theta$ are independent with prior distributions, for $j \in\{0, \ldots, J\}$ and $l \in\{1, \ldots, J\}$,

$$
\Psi_{j} \sim \mathcal{N}\left(\psi_{j}, t_{j}^{2}\right) \quad \text { and } \quad \Phi_{l} \sim \mathcal{N}\left(\phi_{l}, s_{l}^{2}\right),
$$

with prior parameters $\psi_{j}, \phi_{l} \in \mathbb{R}$ and $t_{j}>0, s_{l}>0$.

\section{Remarks.}

- For $V=\operatorname{diag}\left(\tau_{0}^{2} ; \tau_{1}^{2}, \sigma_{1}^{2}, \ldots, \tau_{J}^{2}, \sigma_{J}^{2}\right)$ the model above exactly gives the PIC reserving model from Merz-Wüthrich [5]. In the present paper, we now allow for general covariance matrices $V$ (as long as they are positive definite). In (1.1) below, we give an explicit choice that will be applied to a motor thirdparty liability portfolio.

- The PIC model combines both cumulative payments and incurred losses data to get a unified predictor for the total ultimate claim that is based on both sources of information. Thereby, the model assumption $P_{i, J}=I_{i, J}$ guarantees that the total ultimate claim coincides for claims payments and incurred 
losses data. In particular, we obtain the identities

$$
I_{i, j}=I_{i, j-1} \exp \left\{\zeta_{i, j}\right\}, \quad \text { with initial value } I_{i, 0}=\exp \left\{\zeta_{i, 0}\right\}
$$

and by backward recursion

$$
P_{i, j-1}=P_{i, j} \exp \left\{-\xi_{i, j}\right\}, \quad \text { with initial value } P_{i, J}=I_{i, J}
$$

Note that in comparison to Merz-Wüthrich [5], we have exchanged the role of $I_{i, j}$ and $P_{i, l}$. In the original model of Merz-Wüthrich [5], the resulting claims reserves are completely symmetric in the exchange of $I_{i, j}$ and $P_{i, l}$. If we consider the model with dependence, as in Model Assumptions 1.1 above, it is more natural to use incurred losses $I_{i, J}$ as prior for claims payments $P_{i, l}$. This means that Hertig's log-normal model [4] for $I_{i, j}$ plays the role of the prior for Gogol's claims reserving model [3] for $P_{i, l}$; see also Merz-Wüthrich [5].

- If we have prior (expert) knowledge (as a third information channel) this can be used to design the prior distribution of $\boldsymbol{\Theta}$. If there is no prior knowledge we choose non-informative priors for $\boldsymbol{\Theta}$, that is we let $t_{j}^{2} \rightarrow \infty$ and $s_{l}^{2} \rightarrow \infty$ for $j \in\{0, \ldots, J\}$ and $l \in\{1, \ldots, J\}$.

- The assumption $P_{i, J}=I_{i, J}$ means that all claims are settled after $J$ development years and there is no so-called tail development factor. If there is a claims development beyond development year $J$, then one can extend the PIC model for the estimation of a tail development factor; see Merz-Wüthrich [6] for more details.

- Under Model Assumptions 1.1, the distribution of the ultimate claims $I_{i, J}$ is a priori equal across accident years. However, given the observed data, we observe different posterior distributions for claims of different accident years. Therefore, the PIC reserving method allows for accident year variation (see Theorem 3.2). However, if knowledge about prior differences is available, it should be incorporated in the prior means. This relaxation of the model assumption will still lead to closed-form solutions. A similar effect can be achieved by considering (volume-) adjusted observations.

- Conditional i.i.d. guarantees that we obtain a model of chain ladder type where chain ladder factors do not depend on accident year $i$. Of course, this model assumption requires that the data considered need to be sufficiently regular. If this is not the case, one can introduce prior differences between accident years (see also last bullet point). These more general assumptions still lead to a closed-form solution. The drawback is that the model might become over-parameterized and/or it requires to extended expert knowledge.

- The covariance matrix $V$ allows for modeling dependence within $\Xi_{i}$. In particular, we will choose this covariance matrix such that the correlation between $\zeta_{i, j}$ and $\xi_{i, j}$ is positive because $P_{i, j}$ is contained in $I_{i, j}$ (and hence they are dependent). 
This last bullet point is motivated by the following argument: a positive change from $I_{i, j-1}$ to $I_{i, j}$ means that the claims adjusters increase their expectation in future claims payments. One part of this increased expectation is immediately paid in development period $j$ (and hence contained in both $I_{i, j}$ and $P_{i, j}$ ) and the remaining increased expectation is paid with some settlement delay, which means that we also have higher expectations for $P_{i, l}, l>j$. This argument leads to a following possible explicit choice of the correlation matrix of the random vector $\Xi_{i}$ :

\begin{tabular}{|c||c|c|cc|cc|cc|cc|c|c|c|}
\hline & $\zeta_{i, 0}$ & $\zeta_{i, 1}$ & $\xi_{i, 1}$ & $\zeta_{i, 2}$ & $\xi_{i, 2}$ & $\zeta_{i, 3}$ & $\xi_{i, 3}$ & $\zeta_{i, 4}$ & $\xi_{i, 4}$ & $\cdots$ & $\zeta_{i, J}$ & $\xi_{i, J}$ \\
\hline \hline$\zeta_{i, 0}$ & 1 & 0 & $\rho_{1}$ & 0 & $\rho_{2}$ & 0 & 0 & 0 & 0 & $\cdots$ & 0 & 0 \\
\hline$\zeta_{i, 1}$ & 0 & 1 & $\rho_{0}$ & 0 & $\rho_{1}$ & 0 & $\rho_{2}$ & 0 & 0 & $\cdots$ & 0 & 0 \\
$\xi_{i, 1}$ & $\rho_{1}$ & $\rho_{0}$ & 1 & 0 & 0 & 0 & 0 & 0 & 0 & $\cdots$ & 0 & 0 \\
\hline$\zeta_{i, 2}$ & 0 & 0 & 0 & 1 & $\rho_{0}$ & 0 & $\rho_{1}$ & 0 & $\rho_{2}$ & $\cdots$ & 0 & 0 \\
$\xi_{i, 2}$ & $\rho_{2}$ & $\rho_{1}$ & 0 & $\rho_{0}$ & 1 & 0 & 0 & 0 & 0 & $\cdots$ & 0 & 0 \\
\hline$\zeta_{i, 3}$ & 0 & 0 & 0 & 0 & 0 & 1 & $\rho_{0}$ & 0 & $\rho_{1}$ & $\cdots$ & 0 & 0 \\
$\xi_{i, 3}$ & 0 & $\rho_{2}$ & 0 & $\rho_{1}$ & 0 & $\rho_{0}$ & 1 & 0 & 0 & $\cdots$ & 0 & 0 \\
\hline$\zeta_{i, 4}$ & 0 & 0 & 0 & 0 & 0 & 0 & 0 & 1 & $\rho_{0}$ & $\cdots$ & 0 & 0 \\
$\xi_{i, 4}$ & 0 & 0 & 0 & $\rho_{2}$ & 0 & $\rho_{1}$ & 0 & $\rho_{0}$ & 1 & $\cdots$ & 0 & 0 \\
\hline$\vdots$ & $\vdots$ & $\vdots$ & $\vdots$ & $\vdots$ & $\vdots$ & $\vdots$ & $\vdots$ & $\vdots$ & $\vdots$ & $\ddots$ & $\vdots$ & $\vdots$ \\
\hline$\zeta_{i, J}$ & 0 & 0 & 0 & 0 & 0 & 0 & 0 & 0 & 0 & $\cdots$ & 1 & $\rho_{0}$ \\
$\xi_{i, J}$ & 0 & 0 & 0 & 0 & 0 & 0 & 0 & 0 & 0 & $\cdots$ & $\rho_{0}$ & 1 \\
\hline
\end{tabular}

The rational behind this correlation matrix is that the incurred losses increments $\zeta_{i, j}$ are (positively) correlated to the claims payments increments $\xi_{i, j}$, $\xi_{i, j+1}$ and $\xi_{i, j+2}$ with positive correlations $\rho_{0}, \rho_{1}$ and $\rho_{2}$, respectively. Note that $\zeta_{i, 0}$ plays the special role of the initial value for incurred losses $I_{i, 0}$ (on the log scale), whereas the initial value for claims payments $P_{i, 0}$ (on the log scale) can be defined by $\xi_{i, 0}=\sum_{j=0}^{J} \zeta_{i, j}-\sum_{l=1}^{J} \xi_{i, l}$.

Organization: In Section 2, we derive the ultimate claim predictor for known parameters $\boldsymbol{\Theta}$ (see Corollary 2.2). The model parameter $\boldsymbol{\Theta}$ is estimated in Section 3, leading to the ultimate claim predictor in Theorem 3.2. In Section 4, we calculate the prediction uncertainty in terms of the conditional mean square error of prediction (MSEP; see Theorem 4.1). We apply the PIC method with dependence with three explicit choices of a covariance matrix of the form (1.1) to a motor third-party liability example in Section 5. In Section 6, we present the conclusions. Furthermore, all proofs are provided in the Appendix. 


\section{ULTIMATE CLAIM PREDICTION FOR KNOWN PARAMETERS $\boldsymbol{\Theta}$}

We can work either with the random vector $\Xi_{i} \in \mathbb{R}^{2 J+1}$ (see Model Assumptions 1.1) or with the logarithmized observations given by the random vector

$$
\begin{aligned}
\mathbf{X}_{i}= & \left(\log I_{i, 0}, \log P_{i, 0}, \log I_{i, 1}, \log P_{i, 1}, \ldots, \log I_{i, J-1}, \log P_{i, J-1} ; \log I_{i, J}\right)^{\prime} \\
& \in \mathbb{R}^{2 J+1} .
\end{aligned}
$$

The consideration of $\Xi_{i}$ was easier for the model definition and for the interpretation of the dependence structure; but often it is more straightforward if we directly work with $\mathbf{X}_{i}$ (under the explicit logarithmized cumulative observations). Note that there is a linear one-to-one correspondence between $\boldsymbol{\Xi}_{i}$ and $\mathbf{X}_{i}$, and we denote the (invertible) transformation matrix by $B$. The explicit form of $B$ is given by

$$
B=\left(\begin{array}{rrrrrrrrr}
1 & 0 & 0 & 0 & 0 & \ldots & 0 & 0 & 0 \\
1 & 1 & -1 & 1 & -1 & \ldots & -1 & 1 & -1 \\
1 & 1 & 0 & 0 & 0 & \ldots & 0 & 0 & 0 \\
1 & 1 & 0 & 1 & -1 & \ldots & -1 & 1 & -1 \\
1 & 1 & 0 & 1 & 0 & \ldots & 0 & 0 & 0 \\
& & \vdots & & & \ddots & & \vdots & \\
1 & 1 & 0 & 1 & 0 & \ldots & 0 & 1 & -1 \\
1 & 1 & 0 & 1 & 0 & \ldots & 0 & 1 & 0
\end{array}\right) .
$$

We then obtain the following conditional multivariate Gaussian distribution for $\mathbf{X}_{i}$ :

$$
\left.\mathbf{X}_{i}\right|_{\{\Theta\}}=\left.B \Xi_{i}\right|_{\{\Theta\}} \sim \mathcal{N}\left(\boldsymbol{\mu}=\boldsymbol{\mu}(\boldsymbol{\Theta})=B \Theta, \Sigma=B V B^{\prime}\right) .
$$

Conditionally given the parameters $\Theta$ (or $\mu=\mu(\Theta)$, respectively), the random vector $\mathbf{X}_{i}$ is within the multivariate Gaussian distribution family. Our first aim is to study the conditional distribution of the ultimate claim $P_{i, J}=I_{i, J}$, conditionally given the parameters $\Theta$ and the observations

$$
\mathcal{D}_{J}=\left\{I_{i, j}, P_{i, j}: i+j \leq J, 0 \leq i \leq J, 0 \leq j \leq J\right\}
$$

in the upper paid and incurred triangles (see Figure 1).

For accident years $i \in\{1, \ldots, J\}$, define $n=2 J+1$ and $q=q(i)=2(J-$ $i+1) \in\{2, \ldots, 2 J\}$. At time $J$, we have for accident year $i$ observations (given in the upper triangles $\mathcal{D}_{J}$ )

$$
\mathbf{X}_{i}^{(1)}=\left(\log I_{i, 0}, \log P_{i, 0}, \log I_{i, 1}, \log P_{i, 1}, \ldots, \log I_{i, J-i}, \log P_{i, J-i}\right)^{\prime} \in \mathbb{R}^{q},
$$

and we would like to predict the lower triangles given by

$$
\mathbf{X}_{i}^{(2)}=\left(\log I_{i, J-i+1}, \log P_{i, J-i+1}, \ldots, \log I_{i, J-1}, \log P_{i, J-1} ; \log I_{i, J}\right)^{\prime} \in \mathbb{R}^{n-q} .
$$


This provides, for $i \in\{1, \ldots, J\}$, the following decomposition $\boldsymbol{\mu}=$ $\left(\boldsymbol{\mu}_{i}^{(1)}, \boldsymbol{\mu}_{i}^{(2)}\right)=B \Theta \in \mathbb{R}^{n}$ of the conditional mean:

$$
\mathbb{E}\left[\mathbf{X}_{i}^{(1)} \mid \boldsymbol{\Theta}\right]=\boldsymbol{\mu}_{i}^{(1)}=B_{i}^{(1)} \boldsymbol{\Theta} \in \mathbb{R}^{q} \quad \text { and } \quad \mathbb{E}\left[\mathbf{X}_{i}^{(2)} \mid \boldsymbol{\Theta}\right]=\boldsymbol{\mu}_{i}^{(2)}=B_{i}^{(2)} \boldsymbol{\Theta} \in \mathbb{R}^{n-q},
$$

with partition of $B \in \mathbb{R}^{n \times n}$ given by, for $i \in\{1, \ldots, J\}$,

$$
B=\left(\begin{array}{c}
B_{i}^{(1)} \\
B_{i}^{(2)}
\end{array}\right) \quad \text { with } \quad B_{i}^{(1)} \in \mathbb{R}^{q \times n} \text { and } B_{i}^{(2)} \in \mathbb{R}^{(n-q) \times n} .
$$

In the same way, we also decompose the covariance matrix $\Sigma$ which provides

$$
\Sigma=\left(\begin{array}{cc}
\Sigma_{i}^{(11)} & \Sigma_{i}^{(12)} \\
\Sigma_{i}^{(21)} & \Sigma_{i}^{(22)}
\end{array}\right) \quad \text { with } \quad \Sigma_{i}^{(11)} \in \mathbb{R}^{q \times q} .
$$

For $i=0$, we set $q(0)=n, \mathbf{X}_{0}^{(1)}=\mathbf{X}_{0} \in \mathbb{R}^{n}, \Sigma_{0}^{(11)}=\Sigma$ and $B_{0}^{(1)}=B$.

Having this notation, we provide the prediction of $\mathbf{X}_{i}^{(2)}$, conditionally given $\left\{\boldsymbol{\Theta}, \mathcal{D}_{J}\right\}$ :

Lemma 2.1. Choose an accident year $i \in\{1, \ldots, J\}$. Under Model Assumptions 1.1 we have

$\left.\mathbf{X}_{i}^{(2)}\right|_{\left\{\boldsymbol{\Theta}, \mathcal{D}_{J}\right\}}=\left.\mathbf{X}_{i}^{(2)}\right|_{\left\{\boldsymbol{\Theta}, \mathbf{X}_{i}^{(1)}\right\}} \sim \mathcal{N}\left(\widetilde{\boldsymbol{\mu}}_{i}^{(2)}=\boldsymbol{\mu}_{i}^{(2)}+\Sigma_{i}^{(21)}\left(\Sigma_{i}^{(11)}\right)^{-1}\left(\mathbf{X}_{i}^{(1)}-\boldsymbol{\mu}_{i}^{(1)}\right), \widetilde{\Sigma}_{i}^{(22)}\right)$, with the conditional covariance matrix $\widetilde{\Sigma}_{i}^{(22)}=\Sigma_{i}^{(22)}-\Sigma_{i}^{(21)}\left(\Sigma_{i}^{(11)}\right)^{-1} \Sigma_{i}^{(12)}$.

An immediate consequence of Lemma 2.1 is the following corollary.

Corollary 2.2. Under the assumptions and notations of Lemma 2.1, we obtain for the ultimate claim $I_{i, J}=P_{i, J}$, for $i \in\{1, \ldots, J\}$,

$$
\left.\log I_{i, J}\right|_{\left\{\boldsymbol{\Theta}, \mathcal{D}_{J}\right\}} \sim \mathcal{N}\left(\mathbf{e}_{i}^{\prime} \tilde{\boldsymbol{\mu}}_{i}^{(2)}, \mathbf{e}_{i}^{\prime} \widetilde{\Sigma}_{i}^{(22)} \mathbf{e}_{i}\right),
$$

with $\mathbf{e}_{i}=(0, \ldots, 0,1)^{\prime} \in \mathbb{R}^{n-q}$.

This corollary implies that, conditionally given the parameters $\boldsymbol{\Theta}$ and the observations $\mathcal{D}_{J}$, we get the ultimate claim predictor, for $i \in\{1, \ldots, J\}$,

$$
\mathbb{E}\left[I_{i, J} \mid \boldsymbol{\Theta}, \mathcal{D}_{J}\right]=\exp \left\{\mathbf{e}_{i}^{\prime} \widetilde{\boldsymbol{\mu}}_{i}^{(2)}+\mathbf{e}_{i}^{\prime} \widetilde{\Sigma}_{i}^{(22)} \mathbf{e}_{i} / 2\right\} .
$$

In the special case of a diagonal correlation matrix (1.1), i.e. $\rho_{0}=\rho_{1}=\rho_{2}=0$, this is exactly the predictor derived in Corollary 2.5 of Merz-Wüthrich [5]. 


\section{ESTIMATION OF PARAMETER $\Theta$}

The ultimate claim predictor (2.2) is still based on the unknown parameters $\boldsymbol{\Theta}$, namely

$$
\begin{aligned}
\mathbf{e}_{i}^{\prime} \widetilde{\boldsymbol{\mu}}_{i}^{(2)} & =\mathbf{e}_{i}^{\prime}\left(\boldsymbol{\mu}_{i}^{(2)}+\Sigma_{i}^{(21)}\left(\Sigma_{i}^{(11)}\right)^{-1}\left(\mathbf{X}_{i}^{(1)}-\boldsymbol{\mu}_{i}^{(1)}\right)\right) \\
& =\mathbf{e}_{i}^{\prime}\left(B_{i}^{(2)} \boldsymbol{\Theta}+\Sigma_{i}^{(21)}\left(\Sigma_{i}^{(11)}\right)^{-1}\left(\mathbf{X}_{i}^{(1)}-B_{i}^{(1)} \boldsymbol{\Theta}\right)\right) \\
& =\Gamma_{i} \boldsymbol{\Theta}+\mathbf{e}_{i}^{\prime} \Sigma_{i}^{(21)}\left(\Sigma_{i}^{(11)}\right)^{-1} \mathbf{X}_{i}^{(1)},
\end{aligned}
$$

where we have defined

$$
\Gamma_{i}=\mathbf{e}_{i}^{\prime}\left(B_{i}^{(2)}-\Sigma_{i}^{(21)}\left(\Sigma_{i}^{(11)}\right)^{-1} B_{i}^{(1)}\right) .
$$

In particular, we see that $\mathbf{e}_{i}^{\prime} \widetilde{\boldsymbol{\mu}}_{i}^{(2)}$ is a linear function in $\boldsymbol{\Theta}$. We aim to calculate the posterior distribution of $\boldsymbol{\Theta}$, conditionally given the observations $\mathcal{D}_{J}$. The $\sigma$-field generated by $\mathcal{D}_{J}$ is the same as the one generated by the observations $\widetilde{\mathcal{D}}_{J}=\left\{\mathbf{X}_{0}^{(1)}, \ldots, \mathbf{X}_{J}^{(1)}\right\}$. Therefore, by a slight abuse of notation, we identify the observations $\widetilde{\mathcal{D}}_{J}$ with $\mathcal{D}_{J}$. The likelihood of the logarithmized observations, conditionally given $\Theta$, is then written as, see also (2.1),

$$
l_{\mathcal{D}_{J}}(\Theta) \propto \prod_{i=0}^{J} \exp \left\{-\frac{1}{2}\left(\mathbf{X}_{i}^{(1)}-B_{i}^{(1)} \boldsymbol{\Theta}\right)^{\prime}\left(\Sigma_{i}^{(11)}\right)^{-1}\left(\mathbf{X}_{i}^{(1)}-B_{i}^{(1)} \boldsymbol{\Theta}\right)\right\} .
$$

Under Model Assumptions 1.1, the posterior distribution $u\left(\Theta \mid \mathcal{D}_{J}\right)$ of $\Theta$, given $\mathcal{D}_{J}$, is given by

$$
u\left(\Theta \mid \mathcal{D}_{J}\right) \propto l_{\mathcal{D}_{J}}(\Theta) \exp \left\{-\frac{1}{2}(\boldsymbol{\Theta}-\vartheta)^{\prime} T^{-1}(\Theta-\vartheta)\right\},
$$

where the last term is the prior density of $\Theta$ with prior mean given by

$$
\vartheta=\left(\psi_{0} ; \psi_{1}, \phi_{1}, \psi_{2}, \phi_{2}, \ldots, \psi_{J}, \phi_{J}\right)^{\prime},
$$

and the (diagonal) covariance matrix defined by

$$
T=\operatorname{diag}\left(t_{0}^{2} ; t_{1}^{2}, s_{1}^{2}, t_{2}^{2}, s_{2}^{2}, \ldots, t_{J}^{2}, s_{J}^{2}\right) .
$$

This immediately implies the following lemma.

Lemma 3.1. Under Model Assumptions 1.1, the posterior distribution of $\Theta$, given $\mathcal{D}_{J}$, is a multivariate Gaussian distribution with posterior covariance matrix

$$
T\left(\mathcal{D}_{J}\right)=\left(T^{-1}+\sum_{i=0}^{J}\left(B_{i}^{(1)}\right)^{\prime}\left(\Sigma_{i}^{(11)}\right)^{-1} B_{i}^{(1)}\right)^{-1},
$$


and the posterior mean is given by

$$
\boldsymbol{\vartheta}\left(\mathcal{D}_{J}\right)=T\left(\mathcal{D}_{J}\right)\left[T^{-1} \vartheta+\sum_{i=0}^{J}\left(B_{i}^{(1)}\right)^{\prime}\left(\Sigma_{i}^{(11)}\right)^{-1} \mathbf{X}_{i}^{(1)}\right]
$$

\section{Remarks on credibility theory.}

We define the matrix $S=\left(\sum_{i=0}^{J}\left(B_{i}^{(1)}\right)^{\prime}\left(\Sigma_{i}^{(11)}\right)^{-1} B_{i}^{(1)}\right)^{-1}$, and the credibility weights ( $I$ is the identity matrix)

$$
A=\left(T^{-1}+S^{-1}\right)^{-1} S^{-1} \quad \text { and } \quad I-A=\left(T^{-1}+S^{-1}\right)^{-1} T^{-1},
$$

see also formula (7.11) in Bühlmann-Gisler [2]. This then implies for the posterior covariance matrix $T\left(\mathcal{D}_{J}\right)=A S=(I-A) T$ and we obtain the linear credibility formula for the posterior mean

$$
\vartheta\left(\mathcal{D}_{J}\right)=(I-A) \vartheta+A \mathbf{Y}
$$

with compressed data $\mathbf{Y}=S\left[\sum_{i=0}^{J}\left(B_{i}^{(1)}\right)^{\prime}\left(\Sigma_{i}^{(11)}\right)^{-1} \mathbf{X}_{i}^{(1)}\right]$, see Chapters 7 and 8 in Bühlmann-Gisler [2]. That is, the posterior mean $\vartheta\left(\mathcal{D}_{J}\right)$ is a credibility weighted average between the prior mean $\vartheta$ and the observations $\mathbf{Y}$ with credibility weight $A$.

Theorem 3.2 (PIC predictor). Under Model Assumptions 1.1, we obtain the ultimate claim predictor for $I_{i, J}=P_{i, J}$, given observations $\mathcal{D}_{J}$,

$$
\begin{aligned}
\widehat{I}_{i, J}=\mathbb{E}\left[I_{i, J} \mid \mathcal{D}_{J}\right]= & \exp \left\{\Gamma_{i} \vartheta\left(\mathcal{D}_{J}\right)+\mathbf{e}_{i}^{\prime} \Sigma_{i}^{(21)}\left(\Sigma_{i}^{(11)}\right)^{-1} \mathbf{X}_{i}^{(1)}\right. \\
& \left.+\Gamma_{i} T\left(\mathcal{D}_{J}\right) \Gamma_{i}^{\prime} / 2+\mathbf{e}_{i}^{\prime} \widetilde{\Sigma}_{i}^{(22)} \mathbf{e}_{i} / 2\right\} .
\end{aligned}
$$

Theorem 3.2 gives the ultimate claim predictor that is now based on claims payments, incurred losses and prior expert information. In contrast to MerzWüthrich [5] we can now easily choose any meaningful covariance matrix $V$ for $\Xi_{i}$.

\section{PREDiction UnCERTAinTy}

In order to analyze the prediction uncertainty, we can now study the posterior predictive distribution of $\mathbf{I}_{J}=\left(I_{1, J}, \ldots, I_{J, J}\right)$, which exactly corresponds to the column of unknown ultimate claims, given the observations $\mathcal{D}_{J}$. If $g(\cdot)$ is a sufficiently nice function, we obtain 


$$
\begin{aligned}
\mathbb{E}\left[g\left(\mathbf{I}_{J}\right) \mid \mathcal{D}_{J}\right] & =\int_{\mathbf{x} \in \mathbb{R}^{J}} g(\mathbf{x}) f\left(\mathbf{x} \mid \mathcal{D}_{J}\right) \\
d \mathbf{x} & =\int_{\mathbf{x} \in \mathbb{R}^{J}, \boldsymbol{\Theta}} g(\mathbf{x}) f\left(\mathbf{x} \mid \boldsymbol{\Theta}, \mathcal{D}_{J}\right) u\left(\Theta \mid \mathcal{D}_{J}\right) d \mathbf{x} d \mathbf{\Theta},
\end{aligned}
$$

where $u\left(\Theta \mid \mathcal{D}_{J}\right)$ denotes the posterior density of $\Theta$, given $\mathcal{D}_{J}$. Because the densities $f\left(\mathbf{x} \mid \Theta, \mathcal{D}_{J}\right)$ and $u\left(\Theta \mid \mathcal{D}_{J}\right)$ are explicitly given by Corollary 2.2, Lemma 3.1 and the conditional independence of accident years, given $\boldsymbol{\Theta}$, we can calculate these predictive values $\mathbb{E}\left[g\left(\mathbf{I}_{J}\right) \mid \mathcal{D}_{J}\right]$ numerically, for example using Monte Carlo simulations. This allows for the analysis of any uncertainty and risk measure.

The probably most popular uncertainty measure in actuarial practice that has a closed-form solution is the so-called conditional MSEP. If we consider the total ultimate claim $\sum_{i=1}^{J} I_{i, J}$ and the corresponding predictor $\sum_{i=1}^{J} \widehat{I}_{i, J}$, the conditional MSEP is given by (see Wüthrich-Merz [9], Section 3.1)

$$
\begin{aligned}
& \operatorname{msep}_{\sum_{i=1}^{J} I_{i, J} \mid \mathcal{D}_{J}}\left(\sum_{i=1}^{J} \widehat{I}_{i, J}\right) \\
& =\mathbb{E}\left[\left(\sum_{i=1}^{J} I_{i, J}-\sum_{i=1}^{J} \widehat{I}_{i, J}\right)^{2} \mid \mathcal{D}_{J}\right]=\operatorname{Var}\left(\sum_{i=1}^{J} I_{i, J} \mid \mathcal{D}_{J}\right),
\end{aligned}
$$

where we have used $\widehat{I}_{i, J}=\mathbb{E}\left[I_{i, J} \mid \mathcal{D}_{J}\right]$ in our Bayesian model. Henceforth, we need to calculate this last conditional variance in order to obtain the conditional MSEP.

Theorem 4.1. Under Model Assumptions 1.1, the conditional MSEP is given by

$$
\begin{aligned}
& \operatorname{msep}_{\sum_{i=1}^{J} I_{i, J} \mid \mathcal{D}_{J}}\left(\sum_{i=1}^{J} \widehat{I}_{i, J}\right) \\
& =\sum_{i, k=1}^{J} \widehat{I}_{i, J} \widehat{I}_{k, J}\left(\exp \left\{\Gamma_{i} T\left(\mathcal{D}_{J}\right) \Gamma_{k}^{\prime}+\mathbf{e}_{i}^{\prime} \widetilde{\Sigma}_{i}^{(22)} \mathbf{e}_{i} 1_{\{i=k\}}\right\}-1\right),
\end{aligned}
$$

with $\widehat{I}_{i, J}=\mathbb{E}\left[I_{i, J} \mid \mathcal{D}_{J}\right]$ given by Theorem 3.2.

Thus, we obtain a closed-form solution for both, the ultimate claim predictors $\widehat{I}_{i, J}$ and the corresponding prediction errors, measured by the conditional MSEP. 


\section{MOTOR THIRD-PARTY LIABILITY EXAMPLE}

We apply the PIC model with dependence to the motor third-party liability data given in Tables 4 and 5. In Model Assumptions 1.1, we work with logarithmized paid claims ratios $\zeta_{i, k}$ and logarithmized incurred losses ratios $\xi_{i, k}$, respectively. That means that we have to transform the data in Tables 4 and 5 and we will use in our following calculations logarithmized ratios $\zeta_{i, k}$ for paid claimants and $\xi_{i, k}$ for incurred losses as assumed in Model Assumptions 1.1. Due to the fact that there is no expert knowledge for the specific choice of the means in the prior distributions, we choose in Model Assumptions 1.1 non-informative priors for $\Psi_{l}$ and $\Phi_{j}$, i.e. we let $t_{j}^{2} \rightarrow \infty$ and $s_{l}^{2} \rightarrow \infty$. This implies asymptotically for the credibility weight $A=I$ in Lemma 3.1 and no prior knowledge of the prior means $\phi_{j}$ and $\psi_{l}$ of the prior distributions $\Phi_{j}$ and $\Psi_{l}$ is used in our calculations.

For Model Assumptions 1.1, it remains to choose a suitable covariance matrix $V$. Here we present three different choices of correlation matrices of the type (1.1), which are motivated by an ad hoc estimate for the correlation matrix given below. This estimator for the correlation matrix of the type (1.1) should be seen as an intuitive proposal of a covariance structure and not as an estimator being optimal in some sense. In Section 5.1, we provide a correlation analysis of the motor third-party liability example motivating suitable correlation choices.

\subsection{Correlation matrix choice}

The choice of the correlation matrix type in formula (1.1) reduces the number of parameters to be estimated in comparison to the estimation of a full correlation matrix. Note that we decided for structure (1.1) by pure expert choice. In formula (1.1), we then have to choose $\rho_{l}$ for $l \in\{0,1,2\}$ as

$$
\rho_{l}=\operatorname{Cor}\left(\zeta_{i, k}, \xi_{i, k+l}\right) \quad \text { for } \quad i=1, \ldots, J \text { and } k=1, \ldots, J-l,
$$

and $\rho_{l}=\operatorname{Cor}\left(\zeta_{i, 0}, \xi_{i, l}\right)$ for $l \in\{1,2\}$. We propose the following ad hoc estimators for $\rho_{l}$ for $l \in\{0,1,2\}$ :

1. For the unknown means $\Psi_{k}=\mathbb{E}\left(\zeta_{i, k}\right), \Phi_{k}=\mathbb{E}\left(\xi_{i, k}\right)$ and variances $\sigma_{\zeta_{k}}^{2}=$ $\operatorname{Var}\left(\zeta_{i, k}\right)$ and $\sigma_{\xi_{k}}^{2}=\operatorname{Var}\left(\xi_{i, k}\right)$, we use sample estimates

$$
\begin{aligned}
& \hat{\Psi}_{k}=\frac{1}{J-k+1} \sum_{i=0}^{J-k} \zeta_{i, k}, \quad k=0, \ldots, J, \\
& \hat{\sigma}_{\zeta_{k}}^{2}=\frac{1}{J-k} \sum_{i=0}^{J-k}\left(\zeta_{i, k}-\hat{\Psi}_{k}\right)^{2}, \quad k=0, \ldots, J-1,
\end{aligned}
$$


and

$$
\begin{aligned}
& \hat{\Phi}_{k}=\frac{1}{J-k+1} \sum_{i=0}^{J-k} \xi_{i, k}, \quad k=1, \ldots, J, \\
& \hat{\sigma}_{\xi_{k}}^{2}=\frac{1}{J-k} \sum_{i=0}^{J-k}\left(\xi_{i, k}-\hat{\Phi}_{k}\right)^{2}, \quad k=1, \ldots, J-1 .
\end{aligned}
$$

Since for the estimation of the last variance parameters $\hat{\sigma}_{\zeta_{J}}^{2}$ and $\hat{\sigma}_{\xi_{J}}^{2}$ there is only one observation in the observed triangle, we use the extrapolation formula

$\hat{\sigma}_{\zeta_{J}}^{2}=\min \left\{\hat{\sigma}_{\zeta_{J-2}}^{2}, \hat{\sigma}_{\zeta_{J-1}}^{2}, \hat{\sigma}_{\zeta_{J-2}}^{4} / \hat{\sigma}_{\zeta_{J-1}}^{2}\right\}$ and $\hat{\sigma}_{\xi_{J}}^{2}=\min \left\{\hat{\sigma}_{\xi_{J-2}}^{2}, \hat{\sigma}_{\xi_{J-1}}^{2}, \hat{\sigma}_{\xi_{J-2}}^{4} / \hat{\sigma}_{\xi_{J-1}}^{2}\right\}$.

2. We consider for accident year $i \in\{0, \ldots, J\}$ the standardized observations $\tilde{\zeta}_{i, k}=\frac{\zeta_{i, k}-\hat{\Psi}_{k}}{\hat{\sigma}_{\zeta_{k}}}$ for $k=0, \ldots J-i, \quad \tilde{\xi}_{i, k}=\frac{\xi_{i, k}-\hat{\Phi}_{k}}{\hat{\sigma}_{\xi_{k}}}$ for $k=1, \ldots J-i$.

3. For $l \in\{0,1,2\}$, we use the standard correlation estimator for $\rho_{l}$, based on the dataset

$$
\left\{\left(\tilde{\zeta}_{i, k}, \tilde{\xi}_{i, k+l}\right) \mid k \in\{1, \ldots, J-l\}, i \in\{1, \ldots, J-k-l\}\right\} .
$$

According to our correlation estimators above, we obtain for $\hat{\rho}_{l}$ with $l=$ $0,1,2,3$ ( $\hat{\rho}_{3}$ is defined analogous to the other cases) as a function of the number of observations out of the set (5.1) (the observations are ordered in the way that we first pick up a development period $k$ and then all accident years $i$ up to $J-k-l$ ) being used for estimating $\rho_{l}$ Figure 2. We see in Figure 2 that the assumption of positive correlatedness between $\zeta_{i, k}$ and $\xi_{i, k+l}$ for $l \in\{0,1,2\}$ is evident. For $l=3$ or higher time lags, the correlation estimation is comparably small (about 5\%) and will therefore be neglected in our following considerations. For the sample estimators, stated above and based on the full data set in (5.1) we obtain:

\begin{tabular}{|c|c|c|c|}
\hline$\hat{\rho}_{0}$ & $\hat{\rho}_{1}$ & $\hat{\rho}_{2}$ & $\hat{\rho}_{3}$ \\
\hline $23 \%$ & $27 \%$ & $28 \%$ & $5 \%$ \\
\hline
\end{tabular}

In order to also get correlation sensitivities, we make three explicit correlation choices (cases 1-3 in Table 1), with the correlation estimation results in (5.2) being a point of orientation for our correlation choice and compare it with the uncorrelated case (case 0) of Merz-Wüthrich [5] (see Table 1). For these correlation choices, we have to check whether the resulting covariance matrix $V$ is positive definite (see Model Assumptions 1.1). To calculate $V$, we use the identity

$$
V=\operatorname{Var}^{1 / 2} \operatorname{Cor}_{l} \operatorname{Var}^{1 / 2}
$$




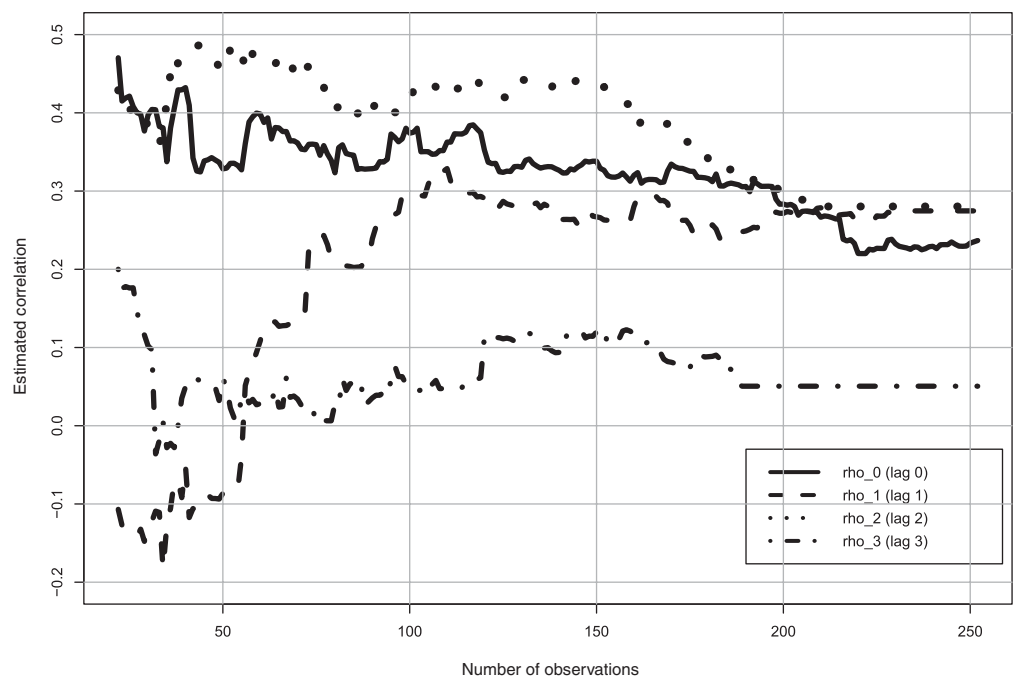

FIGURE 2: Correlation estimators $\hat{\rho}_{l}$ for $\rho_{l}$ for $l \in\{0,1,2,3\}$ as a function of the number of observations used for the estimation.

TABLE 1

INDEPENDENT CASE AND THREE EXPLICIT CHOICES OF CORRELATIONS.

\begin{tabular}{cccc}
\hline \hline Case 0 & Case 1 & Case 2 & Case 3 \\
\hline$\rho_{0}=\rho_{1}=\rho_{2}=0 \%$ & $\rho_{0}=30 \%, \rho_{1}=25 \%$, & $\rho_{0}=30 \%, \rho_{1}=25 \%$, & $\rho_{0}=25 \%, \rho_{1}=25 \%$, \\
& $\rho_{2}=40 \%$ & $\rho_{2}=30 \%$ & $\rho_{2}=30 \%$ \\
\hline \hline
\end{tabular}

where Cor $_{l}$ denotes the correlation matrix in (1.1) for the cases $l \in\{1,2,3\}$ and Var denotes the diagonal matrix with the standard variance estimates of $\left(\zeta_{i, 0} ; \zeta_{i, 1}, \xi_{i, 1}, \zeta_{i, 2}, \xi_{i, 2}, \ldots, \zeta_{i, J}, \xi_{i, J}\right)^{\prime} \in \mathbb{R}^{2 J+1}$ on its diagonal, i.e. Var $=$ $\operatorname{diag}\left(\hat{\sigma}_{\zeta_{0}}^{2} ; \hat{\sigma}_{\zeta_{1}}^{2}, \hat{\sigma}_{\xi_{1}}^{2}, \ldots, \hat{\sigma}_{\zeta_{J}}^{2}, \hat{\sigma}_{\xi_{J}}^{2}\right)$. Since $\operatorname{Var}^{1 / 2}$ is diagonal, we only have to check whether the matrix Cor $\mathrm{C}_{l}$ is positive definite. The eigenvalues of the correlation

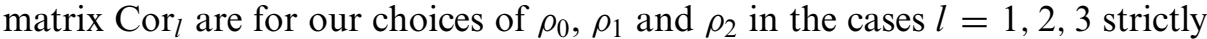
positive, the smallest being $9,2 \times 10^{-12}$ and the largest being $3,8 \times 10^{-03}$ and hence the corresponding covariance matrix $V$ fulfills Model Assumptions 1.1.

Based on these choices for the covariance matrix $V$, we calculate the ultimate claim reserves and the conditional MSEP.

\section{Remarks.}

- We choose by means of explorative data analysis explicit covariance matrices $V$. This was partly done by intuitive expert knowledge.

- In our Bayesian model the derivation of an optimal estimator of the correlation matrix having good statistical properties is not trivial and should be 
subject to more statistical research. Therefore, we present an ad hoc estimator for the correlation matrix and use the resulting estimates as an orientation for our three explicit choices of correlation (cases 1-3).

- Model Assumptions 1.1 allow for arbitrary covariance matrices as long as they are positive definite. If sufficient data for a robust estimation of the $(n(n+1) / 2)$ entries of a general covariance matrix are available, there is no need to reduce to correlations up to lag 2. However, we believe that (due to overparametrization) arbitrary correlation structures are no feasible alternative and expert opinion always needs to specify additional structure.

- Positive definiteness of $V$ should always be checked because most intuitive choices do not always provide a positive definite correlation matrix.

\subsection{Claims reserves and prediction uncertainty}

5.2.1. Claims reserves at time $J$. We consider the expected outstanding loss liabilities (claims reserves)

$$
\hat{R}\left(\mathcal{D}_{J}\right)=E\left[I_{i, J} \mid \mathcal{D}_{J}\right]-P_{i, J-i}
$$

The percental difference between claims reserves with and without dependence is denoted by $D\left(\hat{R}\left(\mathcal{D}_{J}\right)\right)$. We observe in Table 2 that in the first case the claims reserves are about $6 \%$ lower than the claims reserves without dependence. In the other two cases, the difference is still about $3 \%$. This shows that the specific choice of correlation has a crucial impact on the claims reserves. The deeper reason for the higher claims reserves in case 0 is that there has been a slight change in the claims adjuster's philosophy that influences $I_{i, J}$. In cases $1-3$, this change in philosophy is smoothed more over time which should result in more reliable claims reserves.

5.2.2. Prediction uncertainty at time $J$. In Table 3 , we provide the $\mathrm{msep}^{1 / 2}$ for our explicit correlation choices above and for the classical PIC model without dependence (see Table 1). We observe that the prediction uncertainty measured in terms of the conditional MSEP is higher than in the classical PIC model. The reason is that positive correlations in Model Assumptions 1.1 increase the correlations in the ultimate outcomes, and hence the uncertainties. This implies that not considering dependence in the PIC model clearly underestimates the overall uncertainties.

\section{CONCLUSIONS}

In the original PIC model of Merz-Wüthrich [5], a unified predictor of the ultimate claim based on incurred losses and claims payments and the corresponding prediction uncertainty in terms of the conditional MSEP can be derived analytically. The main criticism is that the original PIC model does not allow for modeling dependencies between claims payments and incurred losses as it is observed in the data. 
TABLE 2

CLAIMS RESERVES IN THE CLASSICAL PIC MODEL AND PIC MODEL WITH DEPENDENCE.

\begin{tabular}{crrrrrrr}
\hline \hline $\begin{array}{c}\text { Acc. } \\
\text { year }\end{array}$ & $\begin{array}{r}\hat{R}\left(\mathcal{D}_{J}\right) \\
\text { Case } 0\end{array}$ & $\begin{array}{r}\hat{R}\left(\mathcal{D}_{J}\right) \\
\text { Case 1 }\end{array}$ & $\begin{array}{c}D\left(\hat{R}\left(\mathcal{D}_{J}\right)\right) \\
\text { Case 1 }\end{array}$ & $\begin{array}{r}\hat{R}\left(\mathcal{D}_{J}\right) \\
\text { Case 2 }\end{array}$ & $\begin{array}{r}D\left(\hat{R}\left(\mathcal{D}_{J}\right)\right) \\
\text { Case 2 }\end{array}$ & $\begin{array}{r}\hat{R}\left(\mathcal{D}_{J}\right) \\
\text { Case 3 }\end{array}$ & $\begin{array}{r}D\left(\hat{R}\left(\mathcal{D}_{J}\right)\right) \\
\text { Case 3 }\end{array}$ \\
\hline 1 & 7.726 & 7.729 & $0,0 \%$ & 7.729 & $0,0 \%$ & 7.728 & $0,0 \%$ \\
2 & 12.084 & 12.090 & $0,0 \%$ & 12.089 & $0,0 \%$ & 12.087 & $0,0 \%$ \\
3 & 15.196 & 15.537 & $2,2 \%$ & 15.423 & $1,5 \%$ & 15.397 & $1,3 \%$ \\
4 & 9.916 & 8.291 & $16,4 \%$ & 8.664 & $12,6 \%$ & 8.718 & $12,1 \%$ \\
5 & 20.746 & 21.310 & $2,7 \%$ & 21.169 & $2,0 \%$ & 21.096 & $1,7 \%$ \\
6 & 23.675 & 24.111 & $1,8 \%$ & 24.102 & $1,8 \%$ & 24.047 & $1,6 \%$ \\
7 & 33.328 & 33.410 & $0,2 \%$ & 33.749 & $1,3 \%$ & 33.683 & $1,1 \%$ \\
8 & 35.740 & 37.369 & $4,6 \%$ & 37.327 & $4,4 \%$ & 37.146 & $3,9 \%$ \\
9 & 40.144 & 38.695 & $3,6 \%$ & 39.669 & $1,2 \%$ & 39.767 & $0,9 \%$ \\
10 & 53.888 & 48.764 & $9,5 \%$ & 51.602 & $4,2 \%$ & 51.788 & $3,9 \%$ \\
11 & 62.825 & 59.284 & $5,6 \%$ & 61.134 & $2,7 \%$ & 61.233 & $2,5 \%$ \\
12 & 79.164 & 77.724 & $1,8 \%$ & 78.716 & $0,6 \%$ & 78.352 & $1,0 \%$ \\
13 & 89.437 & 81.510 & $8,9 \%$ & 85.614 & $4,3 \%$ & 85.572 & $4,3 \%$ \\
14 & 88.300 & 79.565 & $9,9 \%$ & 82.942 & $6,1 \%$ & 83.358 & $5,6 \%$ \\
15 & 122.534 & 107.575 & $12,2 \%$ & 115.540 & $5,7 \%$ & 116.508 & $4,9 \%$ \\
16 & 126.151 & 108.955 & $13,6 \%$ & 117.667 & $6,7 \%$ & 118.831 & $5,8 \%$ \\
17 & 126.202 & 119.794 & $5,1 \%$ & 122.695 & $2,8 \%$ & 122.682 & $2,8 \%$ \\
18 & 127.522 & 124.947 & $2,0 \%$ & 126.287 & $1,0 \%$ & 125.897 & $1,3 \%$ \\
19 & 152.078 & 143.847 & $5,4 \%$ & 147.725 & $2,9 \%$ & 148.060 & $2,6 \%$ \\
20 & 185.586 & 170.054 & $8,4 \%$ & 175.798 & $5,3 \%$ & 177.062 & $4,6 \%$ \\
21 & 251.803 & 246.960 & $1,9 \%$ & 248.818 & $1,2 \%$ & 248.554 & $1,3 \%$ \\
Total & 1.664 .045 & 1.567 .522 & $5,8 \%$ & 1.614 .459 & $3,0 \%$ & 1.617 .568 & $2,8 \%$ \\
\hline \hline & & & & & & & \\
\hline
\end{tabular}

TABLE 3

PREDiCTION UNCERTAINTY MSEP ${ }^{1 / 2}$ FOR THE CLASSICAL PIC MODEL AND THE PIC MODEL WITH DEPENDENCE.

\begin{tabular}{lcccc}
\hline \hline & $\begin{array}{c}\text { msep }^{1 / 2} \\
\text { Case 0 }\end{array}$ & $\begin{array}{c}\text { Case }^{1 / 2} \\
\text { Casep }\end{array}$ & $\begin{array}{c}\text { Case 2 }^{1 / 2} \\
\text { Case 3 }\end{array}$ & $\begin{array}{c}\text { Cas }^{1 / 2} \\
\text { Total }\end{array}$ \\
in \% of claims reserves & 40.606 & 48.010 & 49.145 & 48.922 \\
\hline \hline
\end{tabular}

In this paper, we generalize the original PIC model so that it allows for modeling dependence between claims payments and incurred losses data. This is motivated by the fact that, on the one hand, claims payments are contained in incurred losses data and, on the other hand, incurred losses contain additional information, which influences future claims payments data. The data in our example (see Tables 4 and 5) confirm this hypothesis of the dependence between claims payments and incurred losses data (see Figure 2). 
We have seen in the sensitivity analysis that dependence modeling in the PIC method has a crucial impact on the claims reserves and the corresponding MSEPs (see Tables 2 and 3). Note that the classical PIC model of MerzWüthrich [5] underestimates the prediction uncertainty (see Table 5), due to the missing dependence in its structure within accident years. Furthermore, for a better understanding of the influence of prior choices on the reserves and its uncertainty, it might be useful to provide a sensitivity analysis of the method to the choice of priors, which should be subject to an extended case study in future work.

Concluding, the benefits of the PIC method with dependence modeling are that

- two different channels of information are combined to get a unified ultimate loss predictor;

- dependence structures between paid and incurred data can be modeled appropriately;

- prior expert knowledge can be used to design the prior distributions of the parameter vector $\boldsymbol{\Theta}$, otherwise we can choose non-informative priors for $\boldsymbol{\Theta}$. Prior expert opinion should also be used for the design of appropriate correlation structures;

- we can calculate the ultimate claim and the conditional MSEP analytically;

- the full predictive distribution can be derived via Monte Carlo simulations. This allows for the calculation of any risk measure like value-at-risk or expected shortfall.

\section{ACKNOWLEDGEMENT}

The authors would like to thank the anonymous referees for their constructive remarks that have helped to improve earlier versions of this paper.

\section{REFERENCES}

[1] Boyd, S. and VAndenberghe, L. (2004) Convex Optimization. Cambridge: Cambridge University Press.

[2] Bühlmann, H. and Gisler, A. (2005) A Course in Credibility Theory and its Applications. Springer, Berlin.

[3] GogOL, D. (1993) Using expected loss ratios in reserving. Insurance Mathematics \& Economics, 12(3), 297-299.

[4] Hertig, J. (1985) A statistical approach to the IBNR-reserves in marine insurance. Astin Bulletin, 15(2), 171-183.

[5] Merz, M. and WÜthrich, M.V. (2010) Paid-incurred chain claims reserving method. Insurance Mathematics \& Economics, 46(3), 568-579.

[6] Merz, M. and WÜTHRICH, M.V. (2010) Estimation of tail factors in the paid-incurred chain reserving method (preprint).

[7] Posthuma, B., CAtor, E.A., Veerkamp, W. and Zwet, van E.W. (2008) Combined analysis of paid and incurred losses. CAS E-Forum Fall, 2008, 272-293.

[8] QUARG, G. and MACK, T. (2004) Munich chain ladder. Blätter DGVFM, 26, 597-630. 
[9] Wüthrich, M.V. and Merz, M. (2008) Stochastic Claims Reserving Methods in Insurance. Wiley.

SEBASTIAN HAPP

Department of Business Administration, University of Hamburg, 20146 Hamburg, Germany

MARIO V. WÜTHRICH

Department of Mathematics, ETH Zurich, RiskLab, 8092 Zurich, Switzerland

\section{APPENDIX}

\section{APPENDIX. PROOFS}

In this appendix, we prove all the statements.

Proof of Lemma 2.1. Conditionally given the parameters $\boldsymbol{\Theta}$, we have that $\boldsymbol{\Xi}_{i}$ for different accident years $i$ are independent and hence so are $\mathbf{X}_{i}$. Therefore, conditionally given the parameters $\Theta$ and $\mathcal{D}_{J}$, only the observations with accident year $i$ within $\mathcal{D}_{J}$ are relevant for the study of the distribution of $\mathbf{X}_{i}$. This proves the first equality of the claim in Lemma 2.1. The second distributional claim is then a well-known result for multivariate Gaussian distributions. For the Schur complement of $\Sigma_{i}^{(11)}$ in $\Sigma$, we also refer to Section A.5.5 in BoydVandenberghe [1]. This proves the lemma.

Proof of Lemma 3.1. From (3.2) it immediately follows that the posterior distribution of $\boldsymbol{\Theta}$, given $\mathcal{D}_{J}$, is a multivariate Gaussian distribution. The first and second moments are then found by squaring out all terms.

Proof of Theorem 3.2. Using the tower property for conditional expectations, we obtain from formulas (2.2) and (3.2):

$$
\begin{aligned}
\mathbb{E}\left[I_{i, J} \mid \mathcal{D}_{J}\right] & =\mathbb{E}\left[\mathbb{E}\left[I_{i, J} \mid \boldsymbol{\Theta}, \mathcal{D}_{J}\right] \mid \mathcal{D}_{J}\right] \\
& =\mathbb{E}\left[\exp \left\{\Gamma_{i} \boldsymbol{\Theta}\right\} \mid \mathcal{D}_{J}\right] \exp \left\{\mathbf{e}_{i}^{\prime} \Sigma_{i}^{(21)}\left(\Sigma_{i}^{(11)}\right)^{-1} \mathbf{X}_{i}^{(1)}+\mathbf{e}_{i}^{\prime} \widetilde{\Sigma}_{i}^{(22)} \mathbf{e}_{i} / 2\right\} .
\end{aligned}
$$

But then the proof is a consequence from Lemma 3.1.

Proof of Theorem 4.1. With the variance decoupling formula, we obtain

$$
\begin{aligned}
& \operatorname{Var}\left(\sum_{i=1}^{J} I_{i, J} \mid \mathcal{D}_{J}\right)=\sum_{i, k=1}^{J} \operatorname{Cov}\left(I_{i, J}, I_{k, J} \mid \mathcal{D}_{J}\right) \\
& =\sum_{i, k=1}^{J} \operatorname{Cov}\left(\mathbb{E}\left[I_{i, J} \mid \Theta, \mathcal{D}_{J}\right], \mathbb{E}\left[I_{k, J} \mid \Theta, \mathcal{D}_{J}\right] \mid \mathcal{D}_{J}\right)+\sum_{i=1}^{J} \mathbb{E}\left[\operatorname{Var}\left(I_{i, J} \mid \Theta, \mathcal{D}_{J}\right) \mid \mathcal{D}_{J}\right]
\end{aligned}
$$


where for the second term we have used the conditional independence of different accident years, given $\Theta$. Thus, we need to calculate these last two terms. Using Corollary 2.2 and (3.2),

$$
\mathbb{E}\left[I_{i, J} \mid \boldsymbol{\Theta}, \mathcal{D}_{J}\right]=\exp \left\{\Gamma_{i} \boldsymbol{\Theta}+\mathbf{e}_{i}^{\prime} \Sigma_{i}^{(21)}\left(\Sigma_{i}^{(11)}\right)^{-1} \mathbf{X}_{i}^{(1)}+\mathbf{e}_{i}^{\prime} \widetilde{\Sigma}_{i}^{(22)} \mathbf{e}_{i} / 2\right\}
$$

and

$$
\operatorname{Var}\left(I_{i, J} \mid \boldsymbol{\Theta}, \mathcal{D}_{J}\right)=\mathbb{E}\left[I_{i, J} \mid \boldsymbol{\Theta}, \mathcal{D}_{J}\right]^{2}\left(\exp \left\{\mathbf{e}_{i}^{\prime} \widetilde{\Sigma}_{i}^{(22)} \mathbf{e}_{i}\right\}-1\right) .
$$

We first treat the second term. It provides

$$
\begin{aligned}
\mathbb{E} & {\left[\operatorname{Var}\left(I_{i, J} \mid \boldsymbol{\Theta}, \mathcal{D}_{J}\right) \mid \mathcal{D}_{J}\right] } \\
& =\mathbb{E}\left[\exp \left\{2 \Gamma_{i} \boldsymbol{\Theta}+2 \mathbf{e}_{i}^{\prime} \Sigma_{i}^{(21)}\left(\Sigma_{i}^{(11)}\right)^{-1} \mathbf{X}_{i}^{(1)}+\mathbf{e}_{i}^{\prime} \widetilde{\Sigma}_{i}^{(22)} \mathbf{e}_{i}\right\} \mid \mathcal{D}_{J}\right]\left(\exp \left\{\mathbf{e}_{i}^{\prime} \widetilde{\Sigma}_{i}^{(22)} \mathbf{e}_{i}\right\}-1\right) \\
& =\mathbb{E}\left[I_{i, J} \mid \mathcal{D}_{J}\right]^{2} \exp \left\{\Gamma_{i} T\left(\mathcal{D}_{J}\right) \Gamma_{i}^{\prime}\right\}\left(\exp \left\{\mathbf{e}_{i}^{\prime} \widetilde{\Sigma}_{i}^{(22)} \mathbf{e}_{i}\right\}-1\right) .
\end{aligned}
$$

For the first term we need to consider

$$
\begin{aligned}
\operatorname{Cov} & \left(\mathbb{E}\left[I_{i, J} \mid \boldsymbol{\Theta}, \mathcal{D}_{J}\right], \mathbb{E}\left[I_{k, J} \mid \boldsymbol{\Theta}, \mathcal{D}_{J}\right] \mid \mathcal{D}_{J}\right) \\
= & \exp \left\{\mathbf{e}_{i}^{\prime} \Sigma_{i}^{(21)}\left(\Sigma_{i}^{(11)}\right)^{-1} \mathbf{X}_{i}^{(1)}+\mathbf{e}_{i}^{\prime} \widetilde{\Sigma}_{i}^{(22)} \mathbf{e}_{i} / 2+\mathbf{e}_{k}^{\prime} \Sigma_{i}^{(21)}\left(\Sigma_{i}^{(11)}\right)^{-1} \mathbf{X}_{k}^{(1)}+\mathbf{e}_{k}^{\prime} \widetilde{\Sigma}_{i}^{(22)} \mathbf{e}_{k} / 2\right\} \\
& \times \operatorname{Cov}\left(\exp \left\{\Gamma_{i} \boldsymbol{\Theta}\right\}, \exp \left\{\Gamma_{k} \boldsymbol{\Theta}\right\} \mid \mathcal{D}_{J}\right) .
\end{aligned}
$$

For this last covariance term, we have

$$
\begin{aligned}
\operatorname{Cov}\left(\exp \left\{\Gamma_{i} \boldsymbol{\Theta}\right\}, \exp \left\{\Gamma_{k} \boldsymbol{\Theta}\right\} \mid \mathcal{D}_{J}\right) \\
\quad=\mathbb{E}\left[\exp \left\{\Gamma_{i} \boldsymbol{\Theta}\right\} \mid \mathcal{D}_{J}\right] \mathbb{E}\left[\exp \left\{\Gamma_{k} \boldsymbol{\Theta}\right\} \mid \mathcal{D}_{J}\right]\left(\exp \left\{\Gamma_{i} T\left(\mathcal{D}_{J}\right) \Gamma_{k}^{\prime}\right\}-1\right) .
\end{aligned}
$$

Henceforth, we obtain for the first term

$$
\begin{aligned}
& \operatorname{Cov}\left(\mathbb{E}\left[I_{i, J} \mid \Theta, \mathcal{D}_{J}\right], \mathbb{E}\left[I_{k, J} \mid \Theta, \mathcal{D}_{J}\right] \mid \mathcal{D}_{J}\right) \\
& \quad=\mathbb{E}\left[I_{i, J} \mid \mathcal{D}_{J}\right] \mathbb{E}\left[I_{k, J} \mid \mathcal{D}_{J}\right]\left(\exp \left\{\Gamma_{i} T\left(\mathcal{D}_{J}\right) \Gamma_{k}^{\prime}\right\}-1\right)
\end{aligned}
$$

Collecting all the terms and using $\widehat{I}_{i, J}=\mathbb{E}\left[I_{i, J} \mid \mathcal{D}_{J}\right]$ completes the proof. 
TABLE 4

CUMULATIVE CLAIMS PAYMENTS $P_{i, j}, i+j \leq 21$, DATA FROM A MOTOR THIRD-PARTY LIABILITY EXAMPLE.

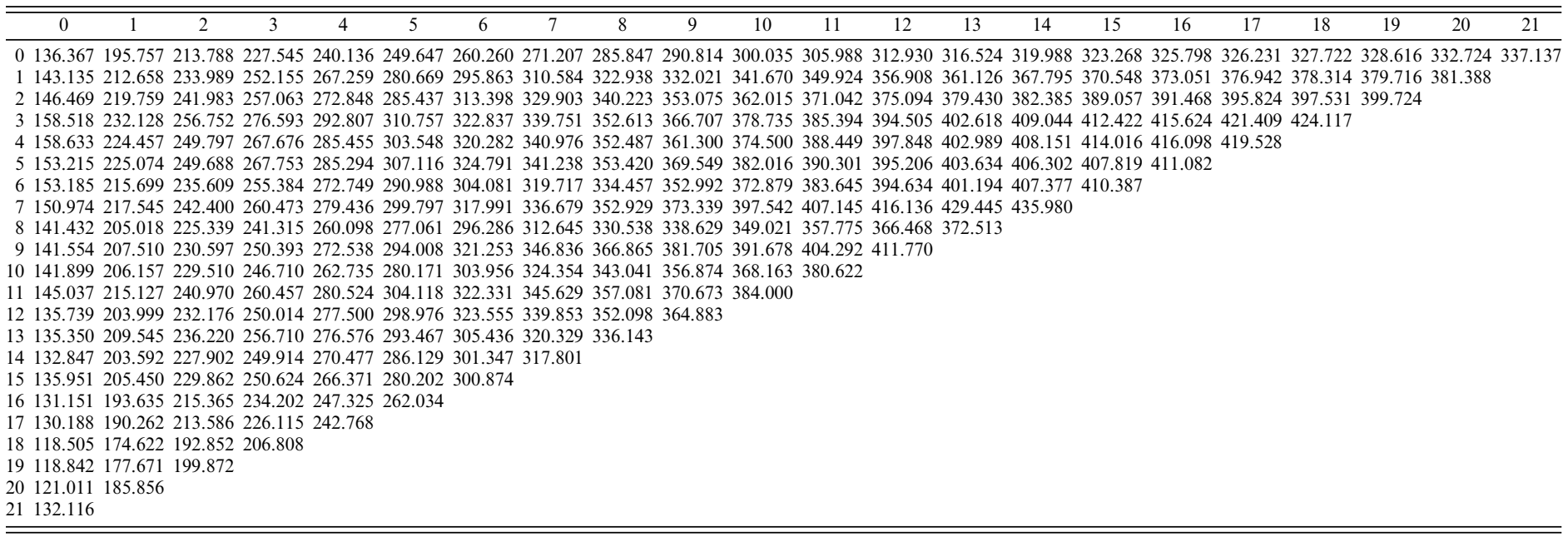


TABLE 5

INCURRED LOSSES $I_{i, j}, i+j \leq 21$, DATA FROM A MOTOR THIRD-PARTY LIABILITY EXAMPLE.

\begin{tabular}{|c|c|c|c|c|c|c|c|c|c|c|c|c|c|c|c|c|c|c|c|c|c|}
\hline 0 & 1 & 2 & 3 & 4 & 5 & 6 & 7 & 8 & 9 & 10 & 11 & 12 & 13 & 14 & 15 & 16 & 17 & 18 & 19 & 20 & 21 \\
\hline 0370.661 & 369.491 & 358.572 & 344.401 & 346.665 & 342.370 & 344.807 & 344.529 & 346.520 & 344.774 & 345.346 & 344.592 & 339.747 & 340.214 & 338.824 & 338.687 & 338.119 & 337.285 & 336.834 & 336.271 & 336.647 & 337.137 \\
\hline 1419.289 & 416.218 & 393.048 & 384.661 & 383.508 & 376.001 & 382.602 & 383.968 & 384.388 & 383.803 & 387.951 & 389.023 & 386.576 & 389.510 & 388.477 & 388.833 & 389.687 & 390.600 & 389.190 & 388.147 & 388.548 & \\
\hline 2437.568 & 427.975 & 415.222 & 407.791 & 406.460 & 418.175 & 411.091 & 406.724 & 410.959 & 413.780 & 414.044 & 410.973 & 410.142 & 409.120 & 410.870 & 414.860 & 412.483 & 410.468 & 410.964 & 410.768 & & \\
\hline 3450.610 & 448.418 & 431.294 & 424.361 & 432.825 & 427.773 & 436.947 & 434.592 & 437.282 & 437.315 & 438.579 & 439.037 & 440.049 & 439.942 & 439.561 & 437.789 & 437.218 & 439.781 & 438.979 & & & \\
\hline 4468.979 & 450.942 & 432.591 & 435.162 & 423.583 & 420.876 & 424.588 & 424.632 & 429.180 & 431.788 & 429.673 & 433.284 & 430.848 & 432.002 & 431.379 & 429.732 & 429.539 & 428.875 & & & & \\
\hline 5443.189 & 434.315 & 433.325 & 425.332 & 424.921 & 422.432 & 421.233 & 422.904 & 424.484 & 426.661 & 431.462 & 431.676 & 434.368 & 433.599 & 431.820 & 432.994 & 433.037 & & & & & \\
\hline 6435.307 & 422.149 & 412.660 & 405.190 & 402.367 & 402.597 & 411.096 & 412.873 & 420.973 & 432.325 & 437.067 & 433.610 & 435.824 & 435.656 & 434.796 & 436.011 & & & & & & \\
\hline 7475.948 & 438.817 & 432.413 & 436.710 & 436.904 & 443.156 & 448.347 & 463.171 & 468.412 & 476.070 & 474.593 & 473.324 & 477.058 & 472.283 & 473.524 & & & & & & & \\
\hline 8447.021 & 422.678 & 405.919 & 399.462 & 400.047 & 398.297 & 406.939 & 404.834 & 409.056 & 411.421 & 412.002 & 410.739 & 409.744 & 413.587 & & & & & & & & \\
\hline 9457.229 & 444.054 & 436.390 & 436.853 & 442.292 & 453.494 & 456.363 & 460.272 & 459.591 & 456.975 & 455.336 & 454.500 & 453.068 & & & & & & & & & \\
\hline 10462.989 & 464.776 & 447.833 & 432.893 & 432.124 & 442.743 & 451.994 & 451.534 & 450.528 & 450.845 & 448.398 & 442.810 & & & & & & & & & & \\
\hline 11484.915 & 468.800 & 454.958 & 447.601 & 461.106 & 470.358 & 465.346 & 468.879 & 461.537 & 456.753 & 453.919 & & & & & & & & & & & \\
\hline 12462.028 & 429.610 & 438.929 & 454.797 & 468.116 & 468.721 & 469.907 & 463.823 & 459.524 & 452.385 & & & & & & & & & & & & \\
\hline 13450.908 & 456.030 & 476.259 & 483.129 & 476.952 & 464.941 & 453.391 & 445.089 & 434.103 & & & & & & & & & & & & & \\
\hline 14426.385 & 428.504 & 456.796 & 449.886 & 445.397 & 432.021 & 412.353 & 402.565 & & & & & & & & & & & & & & \\
\hline 15461.078 & 477.458 & 480.960 & 471.869 & 462.978 & 444.670 & 437.203 & & & & & & & & & & & & & & & \\
\hline 16444.123 & 430.684 & 433.664 & 419.422 & 403.126 & 396.903 & & & & & & & & & & & & & & & & \\
\hline 17433.830 & 407.931 & 393.723 & 371.800 & 361.853 & & & & & & & & & & & & & & & & & \\
\hline $18 \quad 418.202$ & 374.855 & 338.598 & 324.790 & & & & & & & & & & & & & & & & & & \\
\hline 19426.853 & 373.282 & 351.590 & & & & & & & & & & & & & & & & & & & \\
\hline 20410.810 & 394.477 & & & & & & & & & & & & & & & & & & & & \\
\hline 21405.597 & & & & & & & & & & & & & & & & & & & & & \\
\hline
\end{tabular}

